\title{
9 Works Cited
}

\subsection{A. G. Baumgarten}

The works by Baumgarten listed below are cited by the indicated abbreviation with a paragraph or page number. I cite both the original and the translation listed here. All other translations of quoted material are by Anthony Mahler unless otherwise noted. All emphases are in the source text except for when stated otherwise.

AE Baumgarten, Alexander Gottlieb. Aesthetica. 2 vols. Frankfurt an der Oder: Johann Christian Kleyb, 1750/1758. Facsimile, Hildesheim: Georg Olms, 1961.

English translations by Maya Maskarinec and Alexandre Roberts for this publication.

ETH Baumgarten, Alexander Gottlieb. Ethica philosophica. Halle an der Saale: C. H. Hemmerde, 1751. Facsimile, Hildesheim: Georg Olms, 1969.

English translations by Maya Maskarinec and Alexandre Roberts for this publication.

KOLL Baumgarten, Alexander Gottlieb. “Kollegium über die Ästhetik.” In Alexander Gottlieb Baumgarten: Seine Bedeutung und Stellung in der Leibniz-Wolffischen Philosophie und seine Beziehung zu Kant; Nebst Veröffentlichung einer bisher unbekannten Handschrift der Ästhetik Baumgartens by Bernhard Popper. Borna-Leipzig: Robert Noske, 1907. 59-258.

English translations by Anthony Mahler for this publication.

MED Baumgarten, Alexander Gottlieb. Meditationes philosophicae de nonnullis ad poema pertinentibus. Magdeburg: Johann Heinrich Grunert, 1735.

English translations from: Baumgarten, Alexander Gottlieb. Reflections on Poetry: Alexander Gottlieb Baumgarten's “Meditationes philosophicae de nonnullis ad poema pertinentibus.” Trans. Karl Aschenbrenner and William B. Holther. Berkeley: University of California Press, 1954.

MET Baumgarten, Alexander Gottlieb. Metaphysica. 7th ed. Halle an der Saale: C. H. Hemmerde, 1779. Facsimile, Hildesheim: Georg Olms, 2012.

English translations from: Baumgarten, Alexander Gottlieb. Metaphysics: A Critical Translation with Kant's Elucidations, Selected Notes, and Related Materials. Trans. [based on the 4th ed., 1757] Courtney D. Fugate and John Hymers. London: Bloomsbury, 2014.

MET1 Baumgarten, Alexander Gottlieb. Metaphysica. Halle an der Saale: C. H. Hemmerde, 1739.

English translations by Maya Maskarinec and Alexandre Roberts for this publication.

PHB [Baumgarten, Alexander Gottlieb]. Philosophische Brieffe von Aletheophilus. Frankfurt an der Oder, 1741.

English translations by Anthony Mahler for this publication.

PHG Baumgarten, Alexander Gottlieb. Philosophia generalis. Ed. Johann Christian Förster. Halle an der Saale: C. H. Hemmerde, 1770. Facsimile, Hildesheim: Georg Olms, 1968.

○ OpenAccess. (c) 2020 Frauke Berndt, published by De Gruyter. (cc) BY-NC-ND This work is licensed under the Creative Commons Attribution-NonCommercial-NoDerivatives 4.0 License. 


\subsection{Primary Sources}

Abbt, Thomas. "Leben und Charakter Alexander Gottlieb Baumgartens.” In Vermischte Werke. Vol. 4. Berlin: Friedrich Nicolai, 1780. Facsimile, Hildesheim: Georg Olms, 1978. 213-244.

Adelung, Johann Christoph. Grammatisch-kritisches Wörterbuch der Hochdeutschen Mundart, mit beständiger Vergleichung der übrigen Mundarten, besonders aber der Oberdeutschen. 2nd ed. 4 vols. Leipzig: Johann Gottlob Immanuel Breitkopf, 1793-1801. Agamben, Giorgio. Homo sacer: Sovereign Power and Bare Life. Trans. Daniel Heller-Roazen. Stanford: Stanford University Press, 1998.

Aristotle. De interpretatione. In The Complete Works of Aristotle: The Revised Oxford Translation. Ed. Jonathan Barnes. Vol. 1. Princeton: Princeton University Press, 1984. 25-38.

Aristotle. On Divination through Sleep. In On Sleep and Dreams. Trans. David Gallop. Warminster: Aris \& Phillips, 1996. 106-118.

Aristotle. On Rhetoric: A Theory of Civic Discourse. Trans. George A. Kennedy. 2nd ed. New York: Oxford University Press, 2007.

Aristotle. Prior Analytics. Trans. Robin Smith. Indianapolis: Hackett, 1989.

Babrius and Phaedrus. Fables. Trans. Ben Edwin Perry. Loeb Classical Library 436. Cambridge: Harvard University Press, 1965.

Bleuler, Eugen. “Die Ambivalenz.” In Universität Zürich: Festgabe zur Einweihung der Neubauten 18. April 1914. Zurich: Schulthess, 1914. 93-106.

Bloom, Harold. The Anxiety of Influence: A Theory of Poetry. 2nd ed. New York: Oxford University Press, 1997.

Bouhours, Dominique. La manière de bien penser dans les ouvrages d'esprit. Paris: Veuve de S. Mabre-Cramoisy, 1688. Facsimile, Hildesheim: Georg Olms, 1974.

Breitinger, Johann Jakob. Critische Dichtkunst. Zurich: Conrad Orell, 1740. Facsimile, Stuttgart: Metzlersche Verlagsbuchhandlung, 1966.

Cassirer, Ernst. "The Concept of Symbolic Form in the Construction of the Human Sciences (1923)." In The Warburg Years, 72-100.

Cassirer, Ernst. "Einleitung." In Neue Abhandlungen über den menschlichen Verstand by Gottfried Wilhelm Leibniz. Trans. Cassirer. Vol. 3 of Philosophische Werke. Ed. Cassirer. Hamburg: Felix Meiner, 1996. vi-xxxi.

Cassirer, Ernst. The Philosophy of Symbolic Forms. Vol. 1, Language. Trans. Ralph Manheim. New Haven: Yale University Press, 1955.

Cassirer, Ernst. "The Problem of the Symbol and Its Place in the System of Philosophy (1927)." In The Warburg Years, 254-271.

Cassirer, Ernst. The Warburg Years (1919-1933): Essays on Language, Art, Myth, and Technology. Trans. S. G. Lofts and A. Calcagno. New Haven: Yale University Press, 2013.

Catullus, Gaius Valerius. The Poems of Catullus: A Bilingual Edition. Trans. Peter Green. Berkeley: University of California Press, 2005.

Cicero. De inventione, De optimo genere oratorum, Topica. Trans. H. M. Hubbell. Loeb Classical Library 386. Cambridge: Harvard University Press, 1949.

de Man, Paul. “Autobiography as De-facement.” MLA 94.5 (1979): 919-930.

de Man, Paul. "The Rhetoric of Temporality." In Blindness and Insight: Essays in the Rhetoric of Contemporary Criticism. 2nd rev. ed. London: Routledge, 2005. 187-228.

Deleuze, Gilles. Difference and Repetition. Trans. Paul Patton. London: Bloomsbury, 2013. 
Derrida, Jacques. “The Double Session.” In Dissemination. Trans. Barbara Johnson. Chicago: University of Chicago Press, 1981.

Derrida, Jacques. "White Mythology: Metaphor in the Text of Philosophy." In Margins of Philosophy. Trans. Alan Bass. Chicago: University of Chicago Press, 1982. 207-271.

Diderot, Denis. Salons. Ed. Jean Seznec and Jean Adhémar. Vol. 3, 1767. Oxford: Oxford University Press, 1963.

Dubos, Jean-Baptiste. Reflexion critiques sur la poésie et sur la peinture. Vol. 1. Paris: Jean Mariette, 1719.

Fineman, Joel. "The Structure of Allegorical Desire." In Allegory and Representation. Ed.

Stephen J. Greenblatt. Baltimore: Johns Hopkins University Press, 1981. 26-60.

Forster, Edward Morgan. Aspects of the Novel. New York: Harcourt, Brace, 1927.

Foucault, Michel. Fearless Speech. Ed. Joseph Pearson. Los Angeles: Semiotext(e), 2001.

Foucault, Michel. The Order of Things: An Archaeology of the Human Sciences. London: Routledge, 2005.

Freud, Sigmund. "The Interpretation of Dreams." In The Standard Edition of the Complete Psychological Works of Sigmund Freud. Ed. and trans. James Strachey in collaboration with Anna Freud. Vol. 5, The Interpretation of Dreams (Second Part) and On Dreams (1900-1901). London: Hogarth Press, 1964.

Frey, Hans-Jost. Vier Veränderungen über Rhythmus. Basel: Engeler, 2000.

Gadamer, Hans-Georg. Truth and Method. Ed. and trans. Garrett Barden and John Cumming. New York: Crossroad, 1975.

Genette, Gérard. Narrative Discourse: An Essay in Method. Trans. Jane E. Lewin. Ithaca: Cornell University Press, 1980.

Genette, Gérard. Narrative Discourse Revisited. Trans. Jane E. Lewin. Ithaca: Cornell University Press, 1988.

Genette, Gérard. Palimpsests: Literature in the Second Degree. Trans. Channa Newman and Claude Doubinsky. Lincoln: University of Nebraska Press, 1997.

Grimm, Jacob, and Wilhelm Grimm. Deutsches Wörterbuch. 16 vols. Leipzig: Hirzel, $1854-1961$.

Hamburger, Käte. The Logic of Literature. Trans. Marilynn J. Rose. 2nd ed. Bloomington: Indiana University Press, 1973.

Herder, Johann Gottfried. "Critical Forests: Fourth Grove, on Riedel's Theory of the Beaux Arts." In Selected Writings on Aesthetics. Ed. and trans. Gregory Moore. Princeton: Princeton University Press, 2006. 177-290.

Herder, Johann Gottfried. "Von Baumgartens Denkart in seinen Schriften.” In Werke. Ed. Martin Bollacher, Jürgen Brummack, Ulrich Gaier, Gunter E. Grimm, Hans Dietrich Irmscher, Rudolf Smend, and Johannes Wallmann. Vol. 1, Frühe Schriften 1764-1772. Ed. Gaier. Frankfurt am Main: Deutscher Klassiker Verlag, 1985. 653-676.

Home, Henry (Lord Kames). Elements of Criticism. 3 vols. Edinburgh, 1762. Facsimile, Hildesheim: Georg Olms, 1970.

Jakobson, Roman. Language in Literature. Ed. Krystyna Pomorska and Stephen Rudy. Cambridge: Belknap Press of Harvard University Press, 1987.

Jakobson, Roman. "Linguistics and Poetics." In Language in Literature, 62-94.

Jakobson, Roman. "On Realism in Art." In Language in Literature, 19-27.

Kant, Immanuel. Critique of the Power of Judgment. Ed. Paul Guyer. Trans. Paul Guyer and Eric Matthews. Cambridge: Cambridge University Press, 2000. 
Leibniz, Gottfried Wilhelm. "Meditations on Knowledge, Truth, and Ideas (1684)." In Philosophical Essays. Ed. and trans. Roger Ariew and Daniel Garber. Indianapolis: Hackett, 1989. 23-27.

Leibniz, Gottfried Wilhelm. New Essays on Human Understanding. Ed. and trans. Peter Remnant and Jonathan Bennett. Cambridge: Cambridge University Press, 1996.

Lessing, Gotthold Ephraim. Laocoön: An Essay on the Limits of Painting and Poetry. Trans. Edward Allen McCormick. Baltimore: Johns Hopkins University Press, 1984.

Lewis, David. On the Plurality of Worlds. Oxford: Basil Blackwell, 1986.

Livy. History of Rome. Trans. B. O. Foster. Vol. 1. Loeb Classical Library 114. Cambridge: Harvard University Press, 1919.

Lucretius. The Nature of Things. Trans. A. E. Stallings. London: Penguin Books, 2007.

Meier, Georg Friedrich. Anfangsgründe aller schönen Wissenschaften. 2nd ed. 3 vols. Halle an der Saale: C. H. Hemmerde, 1754-1759. Facsimile, Hildesheim: Georg Olms, 1976.

Meier, Georg Friedrich. Vertheidigung der Baumgartischen Erklärung eines Gedichts, wider das 5 Stück des 1 Bandes des neuen Büchersaals der schönen Wissenschaften und freyen Künste. Halle an der Saale: C. H. Hemmerde, 1746.

Mendelssohn, Moses. Philosophical Writings. Ed. and trans. Daniel O. Dahlstrom. Cambridge: Cambridge University Press, 1997.

Merleau-Ponty, Maurice. Child Psychology and Pedagogy: The Sorbonne Lectures 1949-1952. Trans. Talia Welsh. Evanston: Northwestern University Press, 2010.

Peirce, Charles Sanders. "The Logic of Quantity." In Collected Papers of Charles Sanders Peirce. Ed. Charles Hartshorne and Paul Weiss. Vol. 4, The Simplest Mathematics. Cambridge: Harvard University Press, 1933. 59-131.

Plato. Phaedrus. Trans. Alexander Nehamas and Paul Woodruff. Indianapolis: Hackett, 1995.

Pliny the Younger. Letters. Trans. Betty Radice. Vol. 1. Loeb Classical Library 55. Cambridge: Harvard University Press, 1969.

Quintilian. The Orator's Education. Ed. and trans. Donald. A. Russell. 5 vols. Loeb Classical Library 124-127, 494. Cambridge: Harvard University Press, 2001.

Rhetorica ad Herennium. Trans. Harry Caplan. Loeb Classical Library 403. Cambridge: Harvard University Press, 1954.

Sartre, Jean-Paul. “Qu'est-ce que la littérature?” Pts. I-VI. Les Temps Modernes 17 (février 1947): 769-805; 18 (mars 1947): 961-988; 19 (avril 1947): 1194-1218; 20 (mai 1947): 1410-1439; 21 (juin 1947): 1607-1641; 22 (juliette 1947): 7-114.

Schatzki, Theodore. "Sayings, Texts and Discursive Formations." In The Nexus of Practices: Connections, Constellations, Practitioners. Ed. Allison Hui, Schatzki, and Elizabeth Shove. Abingdon: Routledge, 2017. 126-140.

Shaftesbury, Third Earl of (Anthony Ashley Cooper). "Sensus Communis, an Essay on the Freedom of Wit and Humour in a Letter to a Friend." In Characteristics of Men, Manners, Opinions, Times. Ed. Lawrence E. Klein. Cambridge: Cambridge University Press, 1999. $29-69$.

Vergil. The Aeneid. Trans. Sarah Ruden. New Haven: Yale University Press, 2008.

Virgil. Eclogues: The Latin Text with a Verse Translation and Brief Notes. Trans. Guy Lee. Liverpool: Francis Cairns, 1980.

Wolff, Christian. Philosophia prima, sive ontologia, methodo scientifica pertractata, qua omnis cognitionis humanae principia continentur. New ed. In Gesammelte Werke. Ed. Jean École. Vol. 2.3. Frankfurt, 1736. Facsimile, Hildesheim: Georg Olms, 1962. 
Wolff, Christian. Psychologia empirica, methodo scientifica pertractata, qua ea, quae de anima humana indubia experientiae fide constant, continentur et ad solidam universae philosophiae practicae ac theologiae naturalis tractationem via sternitur. New rev. ed. In Gesammelte Werke. Ed. Jean École. Vol. 2.5. Frankfurt, 1738. Facsimile, Hildesheim: Georg Olms, 1968.

Zedler, Johann Heinrich. Grosses vollständiges Universal-Lexicon aller Wissenschafften und Künste. 64 vols. Halle an der Saale, 1731-1754.

\subsection{Secondary Sources}

Adler, Hans. "Fundus Animae - der Grund der Seele: Zur Gnoseologie des Dunklen in der Aufklärung." Deutsche Vierteljahrsschrift für Literaturwissenschaft und Geistesgeschichte 62.2 (1988): $197-220$.

Adler, Hans. "Utopie und Imagination: A. G. Baumgartens Fiktionstheorie am Rande der Aufklärung." In Positive Dialektik: Hoffnungsvolle Momente in der deutschen Kultur; Festschrift für Klaus L. Berghahn zum 70. Geburtstag. Ed. Jost Hermand. Bern: Peter Lang, 2007. 17-28.

Adler, Hans, and Lynn L. Wolff, eds. Aisthesis und Noesis: Zwei Erkenntnisformen vom 18. Jahrhundert bis zur Gegenwart. Munich: Wilhelm Fink, 2013.

Aichele, Alexander. "Wahrheit - Gewissheit - Wirklichkeit: Die systematische Ausrichtung von A. G. Baumgartens Philosophie.” In Aichele and Mirbach, “Alexander Gottlieb Baumgarten," 13-36.

Aichele, Alexander, and Dagmar Mirbach, eds. "Alexander Gottlieb Baumgarten: Sinnliche Erkenntnis in der Philosophie des Rationalismus." Thematic issue, Aufklärung 20 (2008).

Albes, Claudia, and Christiane Frey, eds. Darstellbarkeit: Zu einem ästhetisch-philosophischen Problem um 1800. Würzburg: Königshausen \& Neumann, 2003.

Allerkamp, Andrea. "Onirocritica und mundus fabulosus: Traum und Erfindung." In Allerkamp and Mirbach, Schönes Denken, 201-221.

Allerkamp, Andrea, and Dagmar Mirbach, eds. Schönes Denken: A. G. Baumgarten im Spannungsfeld zwischen Ästhetik, Logik und Ethik. Zeitschrift für Ästhetik und Allgemeine Kunstwissenschaft Sonderheft 15. Hamburg: Felix Meiner, 2016.

Aschenbrenner, Karl, and William B. Holther. "Introduction." In Reflections on Poetry: Alexander Gottlieb Baumgarten's “Meditationes philosophicae de nonnullis ad poema pertinentibus." Trans. Aschenbrenner and Holther. Berkeley: University of California Press, 1954. 1- 32.

Baeumler, Alfred. Das Irrationalitätsproblem in der Ästhetik und Logik des 18. Jahrhunderts bis zur Kritik der Urteilskraft. 2nd ed. Tübingen: Max Niemeyer, 1967. Reprint, Darmstadt: Wissenschaftliche Buchgesellschaft, 1981.

Bahr, Petra. Darstellung des Undarstellbaren: Religionstheoretische Studien zum Darstellungsbegriff bei A. G. Baumgarten und I. Kant. Tübingen: Mohr Siebeck, 2004.

Bartsch, Anna-Maria C. Form und Formalismus: Stationen der Ästhetik bei Baumgarten, Kant und Zimmermann. Würzburg: Königshausen \& Neumann, 2017.

Beiser, Frederick C. Diotima's Children: German Aesthetic Rationalism from Leibniz to Lessing. Oxford: Oxford University Press, 2009. 
Bender, Wolfgang. "Rhetorische Tradition und Ästhetik im 18. Jahrhundert: Baumgarten, Meier und Breitinger.” Zeitschrift für Deutsche Philologie 99.4 (1980): 481-506.

Bergmann, Ernst. Die Begründung der deutschen Ästhetik durch Alex. Gottlieb Baumgarten und Georg Friedrich Meier. Leipzig: Röder \& Schunke, 1911.

Berndt, Frauke. "Ex marmore: Evidenz im Ungeformten bei J. J. Winckelmann und A. G. Baumgarten.” In Präsenz und Evidenz fremder Dinge im Europa des 18. Jahrhunderts. Ed. Birgit Neumann. Göttingen: Wallstein, 2015. 73-96.

Berndt, Frauke. "Halle 1735: Die Entdeckung der Literatur." In Medialität: Historische Konstellationen. Ed. Christian Kiening and Martina Stercken. Zurich: Chronos, 2019. 371-377.

Berndt, Frauke. "In the Twilight Zone: Ambiguity and Aesthetics in Baumgarten." In Amphibolie - Ambiguität - Ambivalenz. Ed. Berndt and Stephan Kammer. Würzburg: Königshausen \& Neumann, 2009. 121-136.

Berndt, Frauke. “Die Kunst der Analogie: A. G. Baumgartens literarische Epistemologie.” In Allerkamp and Mirbach, Schönes Denken, 183-199.

Berndt, Frauke. "Mundus poetarum: A. G. Baumgartens Fiktionstheorie." In Komplexität und Einfachheit: DFG-Symposion 2015. Ed. Albrecht Koschorke. Stuttgart: J. B. Metzler, 2017. 316-338.

Berndt, Frauke. Poema / Gedicht: Die epistemische Konfiguration der Literatur um 1750. Berlin: Walter de Gruyter, 2011.

Berndt, Frauke. "Das Reale.” In Handbuch Literatur \& Psychoanalyse. Ed. Berndt and Eckart Goebel. Berlin: Walter de Gruyter, 2017. 638.

Berndt, Frauke. "Rock Sample: Alexander Gottlieb Baumgarten.” In Aesthetic Theory. Ed. Dieter Mersch, Sylvia Sasse, and Sandro Zanetti. Trans. Brian Alkire. Zurich: Diaphanes, 2019. 21-35.

Berndt, Frauke. "Schönes Wollen: A. G. Baumgartens literarische Medienethik." In Campe and Wessels, Bella Parrhesia, 171-194.

Berndt, Frauke. "Symbolisches Wissen: Zur Ökonomie der 'anderen' Logik bei Alexander Gottlieb Baumgarten.” In Kulturen des Wissens im 18. Jahrhundert. Ed. Ulrich Johannes Schneider. Berlin: Walter de Gruyter, 2008. 383-390.

Berndt, Frauke, and Klaus Sachs-Hombach. "Dimensions of Constitutive Ambiguity." In Ambiguity: Language and Communication. Ed. Susanne Winkler. Berlin: Walter de Gruyter, 2015. 271-282.

Blödorn, Andreas, Daniela Langer, and Michael Scheffel. “Einleitung: Stimmen - im Text?" In Stimme(n) im Text: Narratologische Positionsbestimmungen. Ed. Blödorn, Langer, and Scheffel. Berlin: Walter de Gruyter, 2006. 1-8.

Bode, Christoph. "The Aesthetics of Ambiguity." In Actas del XII Congreso Nacional de la Asociación Española de Estudios Anglo-Norteamericanos: Alicante, 19-22 de Diciembre de 1988. Granada: AEDEAN, 1991. 73-83.

Broich, Ulrich, and Manfred Pfister, eds. Intertextualität: Formen, Funktionen, anglistische Fallstudien. Tübingen: Max Niemeyer, 1985.

Buchenau, Stefanie. "Die Einbindung von Poetik und Ästhetik in die Logik der Aufklärung.” In Kunst und Wissen: Beziehungen zwischen Ästhetik und Erkenntnistheorie im 18. und 19. Jahrhundert. Ed. Astrid Bauereisen, Stephan Pabst, and Achim Vesper. Würzburg: Königshausen \& Neumann, 2009. 71-84. 
Buchenau, Stefanie. The Founding of Aesthetics in the German Enlightenment: The Art of Invention and the Invention of Art. Cambridge: Cambridge University Press, 2013.

Buchenau, Stefanie. "Die Sprache der Sinnlichkeit: Baumgartens poetische Begründung der Ästhetik in den Meditationes philosophicae." In Aichele and Mirbach, "Alexander Gottlieb Baumgarten," 151-173.

Campe, Rüdiger. Affekt und Ausdruck: Zur Umwandlung der literarischen Rede im 17. und 18. Jahrhundert. Tübingen: Max Niemeyer, 1990.

Campe, Rüdiger. "Bella Evidentia: Begriff und Figur von Evidenz in Baumgartens Ästhetik." Deutsche Zeitschrift für Philosophie 49.2 (2001): 243-255.

Campe, Rüdiger. “Der Effekt der Form: Baumgartens Ästhetik am Rande der Metaphysik.” In Literatur als Philosophie - Philosophie als Literatur. Ed. Eva Horn, Bettine Menke, and Christoph Menke. Munich: Wilhelm Fink, 2006. 17-33.

Campe, Rüdiger. "Epoche der Evidenz: Knoten in einem terminologischen Netzwerk zwischen Descartes und Kant." In “Intellektuelle Anschauung”: Figurationen von Evidenz zwischen Kunst und Wissen. Ed. Sibylle Peters and Martin Jörg Schäfer. Bielefeld: Transcript, 2006. $25-43$.

Campe, Rüdiger. "Vor Augen Stellen: Über den Rahmen rhetorischer Bildgebung." In Poststrukturalismus: Herausforderung an die Literaturwissenschaft; DFG-Symposion 1995. Ed. Gerhard Neumann. Stuttgart: J. B. Metzler, 1997. 208-225.

Campe, Rüdiger, and Malte Wessels, eds. Bella Parrhesia: Begriff und Figur der freien Rede in der Frühen Neuzeit. Freiburg im Breisgau: Rombach, 2018.

Casula, Mario. La metafisica di A. G. Baumgarten. Milan: Mursia, 1973.

Caygill, Howard. Art of Judgement. Oxford: Basil Blackwell, 1989.

Caygill, Howard. "Über Erfindung und Neuerfindung der Ästhetik.” Deutsche Zeitschrift für Philosophie 49.2 (2001): 233-241.

Croce, Benedetto. "The Aesthetica of Baumgarten." In Philosophy, Poetry, History: An Anthology of Essays. Trans. Cecil Sprigge. London: Oxford University Press, 1966. 427-450.

Croce, Benedetto. Storia dell'estetica per saggi. Bari: Gius. Laterza \& Figli, 1942.

Dockhorn, Klaus. Macht und Wirkung der Rhetorik: Vier Aufsätze zur Ideengeschichte der Vormoderne. Bad Homburg: Gehlen, 1968.

Emmel, Armin. "Logische, ästhetische und metaphysische Wahrheit bei Alexander Gottlieb Baumgarten.” In Identität - Logik - Kritik: Festschrift für Ulrich Pardey zum 65. Geburtstag. Ed. Benedikt Fait and Daniela Zumpf. Berlin: LIT, 2014. 211-242.

Engler, Bernd, and Kurt Müller. "Einleitung: Das Exemplum und seine Funktionalisierungen." In Exempla: Studien zur Bedeutung und Funktion exemplarischen Erzählens. Ed. Engler and Müller. Berlin: Duncker \& Humblot, 1995. 9-20.

Feldman, Karen S. Arts of Connection: Poetry, History, Epochality. Berlin: Walter de Gruyter, 2019.

Finsen, Hans Carl. "Evidenz und Wirkung im ästhetischen Werk Baumgartens: Texttheorie zwischen Philosophie und Rhetorik." Deutsche Vierteljahrsschrift für Literaturwissenschaft und Geistesgeschichte 70.2 (1996): 198-212.

Franke, Ursula. Baumgartens Erfindung der Ästhetik. Münster: Mentis, 2018.

Franke, Ursula. Kunst als Erkenntnis: Die Rolle der Sinnlichkeit in der Ästhetik des Alexander Gottlieb Baumgarten. Wiesbaden: Franz Steiner, 1972. 
Franke, Ursula. "Die Semiotik als Abschluß der Ästhetik: A. G. Baumgartens Bestimmung der Semiotik als ästhetische Propädeutik." Zeitschrift für Semiotik 1.4 (1979): 345-359.

Franke, Ursula. "Sinnliche Erkenntnis - was sie ist und was sie soll: A. G. Baumgartens Ästhetik-Projekt zwischen Kunstphilosophie und Anthropologie." In Aichele and Mirbach, "Alexander Gottlieb Baumgarten," 73-99.

Frey, Christiane. "Zur ästhetischen Übung: Improvisiertes und Vorbewusstes bei A. G. Baumgarten.” In Allerkamp and Mirbach, Schönes Denken, 171-181.

Fuchs, Florian. "Sich des Lügners entzücken: Ästhetische Freiheit und Baumgartens Szene 'schöner' Parrhesie." In Campe and Wessels, Bella Parrhesia. 195-214.

Fugate, Courtney D., and John Hymers. "Introduction." In Fugate and Hymers, Baumgarten and Kant on Metaphysics, 1-4.

Fugate, Courtney D., and John Hymers, eds. Baumgarten and Kant on Metaphysics. Oxford: Oxford University Press, 2018.

Gabriel, Gottfried. "Baumgartens Begriff der 'perceptio praegnans' und seine systematische Bedeutung." In Aichele and Mirbach, "Alexander Gottlieb Baumgarten," 61-71.

Gabriel, Gottfried. "Logik und Rhetorik der Beispiele." In Darstellungsformen der Wissenschaften im Kontrast: Aspekte der Methodik, Theorie und Empirie. Ed. Lutz Danneberg and Jürg Niederhauser. Tübingen: Gunter Narr, 1988. 241-262.

Gaier, Ulrich. "Rhetorisierung des Denkens." In Homo inveniens: Heuristik und Anthropologie am Modell der Rhetorik. Ed. Stefan Metzger and Wolfgang Rapp. Tübingen: Gunter Narr, 2003. 19-31.

Gasché, Rodolphe. "Some Reflections on the Notion of Hypotyposis in Kant." Argumentation 4.1 (1990): 85-100.

Gelley, Alexander. "Introduction.” In Unruly Examples: On the Rhetoric of Exemplarity. Ed. Gelley. Stanford: Stanford University Press, 1995. 1-24.

Gerhardus, Dietfried. "Sprachphilosophie in der Ästhetik." In Philosophy of Language: An International Handbook of Contemporary Research. Ed. Marcelo Dascal, Gerhardus, Kuno Lorenz, and Georg Meggle. Vol. 2. Berlin: Walter de Gruyter, 1996. 1519-1528.

Gess, Nicola. Staunen: Eine Poetik. Göttingen: Wallstein, 2019.

Groddeck, Wolfram. Reden über Rhetorik: Zu einer Stilistik des Lesens. Frankfurt am Main: Stroemfeld, 1995.

Groß, Steffen W. Felix aestheticus: Die Ästhetik als Lehre vom Menschen; Zum 250. Jahrestag des Erscheinens von Alexander Gottlieb Baumgartens "Aesthetica." Würzburg: Königshausen \& Neumann, 2001.

Grote, Simon. The Emergence of Modern Aesthetic Theory: Religion and Morality in Enlightenment Germany and Scotland. Cambridge: Cambridge University Press, 2017. Grote, Simon. "Pietistische Aisthesis und moralische Erziehung bei Alexander Gottlieb Baumgarten.” In Aichele and Mirbach, “Alexander Gottlieb Baumgarten,” 175-198.

Hadot, Pierre. Exercices spirituels et philosophie antique. Paris: Études Augustiniennes, 1981. Hartlaub, Gustav F. "Arcana artis: Spuren alchemistischer Symbolik in der Kunst des 16. Jahrhunderts." Zeitschrift für Kunstgeschichte 6 (1937): 289-324.

Hartlaub, Gustav F. "Signa hermetis: Zwei alte alchemistische Bilderhandschriften." Zeitschrift des deutschen Vereins für Kunstwissenschaft 4 (1937): 93-112, 144-162.

Haverkamp, Anselm. Figura cryptica: Theorie der literarischen Latenz. Frankfurt am Main: Suhrkamp, 2002. 
Haverkamp, Anselm. “Masse mal Beschleunigung: Rhetorik als Meta-Physik der Ästhetik.” In Masse und Medium: Verschiebungen in der Ordnung des Wissens und der Ordnung der Literatur 1800/2000. Ed. Inge Münz-Koenen and Wolfgang Schäffner. Berlin: Akademie Verlag, 2002. 65-77.

Haverkamp, Anselm. "Metaphora dis/continua: Figure in de/construction; Mit einem Kommentar zur Begriffsgeschichte von Quintilian bis Baumgarten.” In Allegorie: Konfigurationen von Text, Bild und Lektüre. Ed. Eva Horn and Manfred Weinberg. Opladen: Westdeutscher Verlag, 1998. 29-45.

Haverkamp, Anselm. "Paradigma Metapher, Metapher Paradigma - Zur Metakinetik hermeneutischer Horizonte (Blumenberg/Derrida, Kuhn/Foucault, Black/White).” In Epochenschwelle und Epochenbewusstsein. Ed. Reinhart Herzog and Reinhart Koselleck. Munich: Wilhelm Fink, 1987. 547-560.

Haverkamp, Anselm. "Die Wiederkehr der Allegorie in der Ästhetik der Avantgarde: Baumgarten in der Vorgeschichte des New Criticism." In Allegorie: DFG-Symposion 2014. Ed. Ulla Haselstein in collaboration with Friedrich Teja Bach, Bettine Menke, and Daniel Selden. Berlin: Walter de Gruyter, 2016. 244-272.

Haverkamp, Anselm. "Wie die Morgenröthe zwischen Nacht und Tag: Alexander Gottlieb Baumgarten und die Begründung der Kulturwissenschaften in Frankfurt an der Oder." Deutsche Vierteljahrsschrift für Literaturwissenschaft und Geistesgeschichte 76.1 (2002): 3-26.

Hees, Johannes. "Denken und Betrachten: Zur Proto-Ästhetik bei Gottfried Wilhelm Leibniz und Barthold Hinrich Brockes." Zeitschrift für Ästhetik und Allgemeine Kunstwissenschaft 64.1 (2019): 87-109.

Heusch, Christine. "Die Ethopoiie in der griechischen und lateinischen Antike: Von der rhetorischen Progymnasma-Theorie zur literarischen Form." In 'HөOחOIIA: La représentation de caractères entre fiction scolaire et réalité vivante à l'époque impériale et tardive. Ed. Eugenio Amato and Jacques Schamp. Salerno: Helios, 2005. 11-33.

Jacob, Joachim. Die Schönheit der Literatur: Zur Geschichte eines Problems von Gorgias bis Max Bense. Tübingen: Max Niemeyer, 2007.

Jäger, Michael. Kommentierende Einführung in Baumgartens "Aesthetica": Zur entstehenden wissenschaftlichen Ästhetik des 18. Jahrhunderts in Deutschland. Hildesheim: Georg Olms, 1980.

Jürgensen, Andreas. "Der ästhetische Horizont: Baumgartens Ästhetik und die Malerei um die Mitte des 18. Jahrhunderts.” Diss., Christian-Albrechts-Universität zu Kiel, 1993.

Kemman, Ansgar. "Evidentia, Evidenz." In Historisches Wörterbuch der Rhetorik. Ed. Gert Ueding. Vol. 2. Tübingen: Max Niemeyer, 1996. Cols. 33-47.

Kinnaman, Ted. “Aesthetics before Kant." In A Companion to Early Modern Philosophy. Ed. Steven Nadler. Malden: Blackwell, 2002. 572-585.

Klibansky, Raymond, Erwin Panofsky, and Fritz Saxl. Saturn and Melancholy: Studies in the History of Natural Philosophy, Religion and Art. London: Thomas Nelson, 1964.

Koch, Peter. "Graphé: Ihre Entwicklung zur Schrift, zum Kalkül und zur Liste." In Schrift, Medien, Kognition: Über die Exteriorität des Geistes. Ed. Koch and Sybille Krämer. Tübingen: Stauffenburg, 1997. 43-81.

Koschorke, Albrecht. Körperströme und Schriftverkehr: Mediologie des 18. Jahrhunderts. 2nd ed. Munich: Wilhelm Fink, 2003. 
Koselleck, Reinhart. Futures Past: On the Semantics of Historical Time. Trans. Keith Tribe. New York: Columbia University Press, 2004.

Krämer, Sybille. "Sprache - Stimme - Schrift: Sieben Gedanken über Performativität als Medialität.” In Performanz: Zwischen Sprachphilosophie und Kulturwissenschaften. Ed. Uwe Wirth. Frankfurt am Main: Suhrkamp, 2003. 323-346.

Krauss, Andrea. "Nuancen des Firmaments: Versuchsanordnungen 'extensiver Klarheit' zwischen Alexander Gottlieb Baumgarten und Barthold Heinrich Brockes." In Allerkamp and Mirbach, Schönes Denken, 223-251.

Kretzmann, Norman. "Syncategoremata, Exponibilia, Sophismata." In The Cambridge History of Later Medieval Philosophy: From the Rediscovery of Aristotle to the Disintegration of Scholasticism, 1100-1600. Ed. Kretzmann, Anthony Kenny, and Jan Pinborg. Cambridge: Cambridge University Press, 1982. 211-245.

Krupp, Anthony. "Cultivation as Maturation: Infants, Children, and Adults in Alexander Gottlieb Baumgarten's Aesthetica." Monatshefte 98.4 (2006): 524-538.

Kurz, Gerhard. Metapher, Allegorie, Symbol. 3rd ed. Göttingen: Vandenhoeck \& Ruprecht, 1993.

Largier, Niklaus. "The Plasticity of the Soul: Mystical Darkness, Touch, and Aesthetic Experience." MLN 125.3 (2010): 536-551.

Linn, Marie-Luise. “A. G. Baumgartens Aesthetica und die antike Rhetorik.” Deutsche Vierteljahrsschrift für Literaturwissenschaft und Geistesgeschichte 41.3 (1967): 424-443.

Lobsien, Eckhard. Wörtlichkeit und Wiederholung: Phänomenologie poetischer Sprache. Munich: Wilhelm Fink, 1995.

Löschner, Claudia. Denksystem: Logik und Dichtung bei Käte Hamburger. Berlin: Ripperger \& Kremers, 2013.

Lyons, John D. Exemplum: The Rhetoric of Example in Early Modern France and Italy. Princeton: Princeton University Press, 1989.

Mahler, Anthony. "Writing Regimens: The Dietetics of Literary Authorship in the Late German Enlightenment." PhD diss., University of Chicago, 2014.

Marcuschi, Luiz Antônio. Die Methode des Beispiels: Untersuchungen über die methodische Funktion des Beispiels in der Philosophie, insbesondere bei Ludwig Wittgenstein. Erlangen: Palm \& Enke, 1976.

Matuschek, Stefan. Über das Staunen: Eine ideengeschichtliche Analyse. Tübingen: Max Niemeyer, 1991.

Meixner, Sebastian. Narratologie und Epistemologie: Studien zu Goethes frühen Erzählungen. Berlin: Walter de Gruyter, 2019.

Menke, Christoph. "Die Disziplin der Ästhetik ist die Ästhetik der Disziplin: Baumgarten in der Perspektive Foucaults.” In Baumgarten-Studien: Zur Geneaologie der Ästhetik. Ed. Rüdiger Campe, Anselm Haverkamp, and Menke. Cologne: August, 2014. 233-247.

Menke, Christoph. Force: A Fundamental Concept of Aesthetic Anthropology. Trans. Gerrit Jackson. New York: Fordham University Press, 2013.

Menke, Christoph. "Schwerpunkt: Zur Aktualität der Ästhetik von Alexander Gottlieb Baumgarten.” Deutsche Zeitschrift für Philosophie 49.2 (2001): 229-231.

Menninghaus, Winfried. “'Darstellung': Friedrich Gottlieb Klopstocks Eröffnung eines neuen Paradigmas." In Was heißt "Darstellen"? Ed. Christiaan L. Hart Nibbrig. Frankfurt am Main: Suhrkamp, 1994. 205-226. 
Menzel, Norbert. “Der anthropologische Charakter des Schönen bei Baumgarten.” Diss., Pontificia Universita Gregoriana, 1969.

Menzer, Paul. "Zur Entstehung von A. G. Baumgartens Ästhetik." Zeitschrift für Deutsche Kulturphilosophie 4 (1938): 288-296.

Mersch, Dieter. "Medial Paradoxes: On Methods of Artistic Production." In Critical Composition Today. Ed. Claus-Steffen Mahnkopf. Hofheim: Wolke, 2006. 62-74.

Mersch, Dieter. "Paradoxien der Verkörperung: Zu einer negativen Semiotik des Symbolischen.” In Aktualität des Symbols. Ed. Frauke Berndt and Christoph Brecht. Freiburg im Breisgau: Rombach, 2005. 33-52.

Mirbach, Dagmar. "Alexander Gottlieb Baumgarten: 'Gnoseologische Rhetorik.”' In Simon, Herders Rhetoriken, 93-111.

Mirbach, Dagmar, ed. Ästhetik by Alexander Gottlieb Baumgarten. Trans. Mirbach. 2 vols. Hamburg: Felix Meiner, 2007.

Mirbach, Dagmar. "Einführung zur fragmentarischen Ganzheit von Alexander Gottlieb Baumgartens Aesthetica (1750/58).” In Ästhetik by Alexander Gottlieb Baumgarten. Trans. Mirbach. Vol. 1. Hamburg: Felix Meiner, 2007. xv-lxxx.

Mirbach, Dagmar. "Ingenium venustum und magnitudo pectoris: Ethische Aspekte von Alexander Gottlieb Baumgartens Aesthetica." In Aichele and Mirbach, "Alexander Gottlieb Baumgarten,” 199-218.

Mirbach, Dagmar. "Magnitudo aesthetica, Aesthetic Greatness: Ethical Aspects of Alexander Gottlieb Baumgarten's Fragmentary Aesthetica (1750/58)." The Nordic Journal of Aesthetics 20.36/37 (2008/2009): 102-128.

Mirbach, Dagmar. "Praeponitur - illustratur: Intertextualität bei A. G. Baumgarten.” In Allerkamp and Mirbach, Schönes Denken, 71-88.

Mirbach, Dagmar, and Andrea Allerkamp. "Ale.theophilus Baumgarten / Wenn die Magd in den Brunnen fällt." In Allerkamp and Mirbach, Schönes Denken, 317-340.

Mülder-Bach, Inka. Im Zeichen Pygmalions: Das Modell der Statue und die Entdeckung der "Darstellung" im 18. Jahrhundert. Munich: Wilhelm Fink, 1998.

Nannini, Alessandro. "Alexander G. Baumgarten and the Lost Letters of Aletheophilus. Notes on a Mystery at the Origins of Modern Aesthetics." Diciottesimo Secolo 2 (2017): 23-43.

Niehues-Pröbsting, Heinrich. "Rhetorische und idealistische Kategorien der Ästhetik." In Kolloquium Kunst und Philosophie. Ed. Willi Oelmüller. Vol. 1, Ästhetische Erfahrung. Paderborn: Ferdinand Schöningh, 1981. 94-110.

Norton, Robert E. The Beautiful Soul: Aesthetic Morality in the Eighteenth Century. Ithaca: Cornell University Press, 1995.

Nuzzo, Angelica. "Determination, Determinability, and the Structure of Ens: Baumgarten's Ontology and Beyond." In Fugate and Hymers, Baumgarten and Kant on Metaphysics, $23-41$.

Oesterreicher, Wulf. “Grenzen der Arbitrarietät: Zum Verhältnis von Laut und Schrift.” In Mimesis und Simulation. Ed. Andreas Kablitz and Gerhard Neumann. Freiburg im Breisgau: Rombach, 1998. 211-233.

Paetzold, Heinz. Ästhetik des deutschen Idealismus: Zur Idee ästhetischer Rationalität bei Baumgarten, Kant, Schelling, Hegel und Schopenhauer. Wiesbaden: Franz Steiner, 1983.

Paetzold, Heinz. "Rhetorik-Kritik und Theorie der Künste in der philosophischen Ästhetik von Baumgarten bis Kant." In Von der Rhetorik zur Ästhetik: Studien zur Entstehung der modernen Ästhetik im 18. Jahrhundert. Ed. Gérard Raulet. Rennes: Philia, 1995. 9-40. 
Patey, Douglas Lane. Probability and Literary Form: Philosophic Theory and Literary Practice in the Augustan Age. Cambridge: Cambridge University Press, 1984.

Peters, Hans Georg. Die Ästhetik Alexander Gottlieb Baumgartens und ihre Beziehungen zum Ethischen. Berlin: Junker und Dünnhaupt, 1934.

Pfister, Manfred. “Konzepte der Intertextualität.” In Broich and Pfister, Intertextualität, 11-20. Pfister, Manfred. "Zur Systemreferenz." In Broich and Pfister, Intertextualität, 52-58.

Pimpinella, Pietro. "Veritas aesthetica: Erkenntnis des Individuellen und mögliche Welten." In Aichele and Mirbach, "Alexander Gottlieb Baumgarten," 37-60.

Plett, Heinrich F. Einführung in die rhetorische Textanalyse. 9th ed. Hamburg: Helmut Buske, 2001.

Plett, Heinrich F. Rhetorik der Affekte: Englische Wirkungsästhetik im Zeitalter der Renaissance. Tübingen: Max Niemeyer, 1975.

Rapp, Christof. “Ähnlichkeit, Analogie und Homonymie bei Aristoteles.” Zeitschrift für philosophische Forschung 46.4 (1992): 526-544.

Reiss, Hans. "Die Einbürgerung der Ästhetik in der deutschen Sprache des achtzehnten Jahrhunderts oder Baumgarten und seine Wirkung." Jahrbuch der deutschen Schillergesellschaft 37 (1993): 109-138.

Riemann, Albert. Die Ästhetik Alexander Gottlieb Baumgartens unter besonderer Berücksichtigung der "Meditationes philosophicae de nonnullis ad poema pertinentibus" nebst einer Übersetzung dieser Schrift. Halle an der Saale: Max Niemeyer, 1928.

Ryan, Marie-Laure. Possible Worlds, Artificial Intelligence, and Narrative Theory. Bloomington: Indiana University Press, 1991.

Ryan, Marie-Laure. "Possible Worlds.” In Handbook of Narratology. Ed. Peter Hühn, Jan Christoph Meister, John Pier, and Wolf Schmid. 2nd ed. Vol. 2. Berlin: Walter de Gruyter, 2014. 726-742.

Scheer, Brigitte. "Baumgartens Ästhetik und die Krise der von ihm begründeten Disziplin.” Philosophische Rundschau 22.1/2 (1976): 108-119.

Scheer, Brigitte. Einführung in die philosophische Ästhetik. Darmstadt: Wissenschaftliche Buchgesellschaft, 1997.

Schmidt, Horst-Michael. Sinnlichkeit und Verstand: Zur philosophischen und poetologischen Begründung von Erfahrung und Urteil in der deutschen Aufklärung (Leibniz, Wolff, Gottsched, Bodmer und Breitinger, Baumgarten). Munich: Wilhelm Fink, 1982.

Schmitt, Arbogast. "Die Entgrenzung der Künste durch ihre Ästhetisierung bei Baumgarten.” In Ästhetische Erfahrung im Zeichen der Entgrenzung der Künste: Epistemische, ästhetische und religiöse Formen von Erfahrung im Vergleich. Ed. Gert Mattenklott. Hamburg: Felix Meiner, 2004. 55-71.

Schmude, Michael P. "Licentia." In Historisches Wörterbuch der Rhetorik. Ed. Gert Ueding. Vol. 5. Tübingen: Max Niemeyer, 2001. Cols. 253-258.

Schulte-Sasse, Jochen. "Aesthetic Orientation in a Decentered World." In A New History of German Literature. Ed. David E. Wellbery, Judith Ryan, Hans Ulrich Gumbrecht, Anton Kaes, Joseph Leo Koerner, and Dorothea E. von Mücke. Cambridge: Belknap Press of Harvard University Press, 2004. 350-355.

Schwaiger, Clemens. Alexander Gottlieb Baumgarten - ein intellektuelles Porträt: Studien zur Metaphysik und Ethik von Kants Leitautor. Stuttgart: Frommann-Holzboog, 2011.

Schwaiger, Clemens. "Baumgartens Ansatz einer philosophischen Ethikbegründung." In Aichele and Mirbach, “Alexander Gottlieb Baumgarten," 219-237. 
Schwaiger, Clemens. "Baumgarten's Theory of Freedom: A Contribution to the Wolff-Lange Controversy." In Fugate and Hymers, Baumgarten and Kant on Metaphysics, 42-60.

Schweizer, Hans Rudolf. Ästhetik als Philosophie der sinnlichen Erkenntnis: Eine Interpretation der "Aesthetica" A. G. Baumgartens mit teilweiser Wiedergabe des lateinischen Textes und deutscher Übersetzung. Basel: Schwabe, 1973.

Seel, Martin. Aesthetics of Appearing. Trans. John Farrell. Stanford: Stanford University Press, 2005.

Simon, Ralf. Die Idee der Prosa: Zur Ästhetikgeschichte von Baumgarten bis Hegel mit einem Schwerpunkt bei Jean Paul. Munich: Wilhelm Fink, 2013.

Simon, Ralf. "Petites perceptions und ästhetische Form." In Leibniz in Philosophie und Literatur um 1800. Ed. Wenchao Li and Monika Meier. Hildesheim: Georg Olms, 2016. 203-229.

Simon, Ralf. "Rhetorik in konstitutionstheoretischer Funktion (Leibniz, Baumgarten, Herder)." In Simon, Herders Rhetoriken, 113-127.

Simon, Ralf, ed. Herders Rhetoriken im Kontext des 18. Jahrhunderts: Beiträge zur Konferenz der Internationalen Herder-Gesellschaft Schloss Beuggen nahe Basel. Heidelberg: Synchron, 2014.

Solms, Friedhelm. Disciplina aesthetica: Zur Frühgeschichte der ästhetischen Theorie bei Baumgarten und Herder. Stuttgart: Klett-Cotta, 1990.

Spalinger, Roland. "Ethopoeia: Historische und theoretische Analyse einer rhetorischen Figur." MA thesis, Universität Zürich, 2018.

Stierle, Karlheinz. "Das bequeme Verhältnis: Lessings Laokoon und die Entdeckung des ästhetischen Mediums." In Das Laokoon-Projekt: Pläne einer semiotischen Ästhetik. Ed. Gunter Gebauer. Stuttgart: J. B. Metzler, 1984. 23-58.

Stierle, Karl-Heinz. "Story as Exemplum - Exemplum as Story: On the Pragmatics and Poetics of Narrative Texts." In New Perspectives in German Literary Criticism: A Collection of Essays. Ed. Richard A. Amacher and Victor Lange. Trans. David Henry Wilson et al. Princeton: Princeton University Press, 1979. 389-417.

Stöckmann, Ernst. Anthropologische Ästhetik: Philosophie, Psychologie und ästhetische Theorie der Emotionen im Diskurs der Aufklärung. Tübingen: Max Niemeyer, 2009.

Strube, Werner. "Alexander Gottlieb Baumgartens Theorie des Gedichts." In Dichtungstheorien der deutschen Frühaufklärung. Ed. Theodor Verweyen in collaboration with Hans-Joachim Kertscher. Tübingen: Max Niemeyer, 1995. 1-25.

Thorsen, Bengerd Juul. “Baumgarten's Meditationes as a Commentary on Horace's ‘Ars Poetica."” Philosophica 44 (2014): 9-25.

Thums, Barbara. Aufmerksamkeit: Wahrnehmung und Selbstbegründung von Brockes bis Nietzsche. Munich: Wilhelm Fink, 2008.

Torra-Mattenklott, Caroline. Metaphorologie der Rührung: Ästhetische Theorie und Mechanik im 18. Jahrhundert. Munich: Wilhelm Fink, 2002.

Trop, Gabriel. "Aesthetic Askesis: Aesthetics as a Technology of the Self in the Philosophy of Alexander Baumgarten." Das achtzehnte Jahrhundert 37.1 (2013): 56-73.

Trop, Gabriel. Poetry as a Way of Life: Aesthetics and Askesis in the German Eighteenth Century. Evanston: Northwestern University Press, 2015.

Vogt-Spira, Gregor. "Vox und Littera: Der Buchstabe zwischen Mündlichkeit und Schriftlichkeit in der grammatischen Tradition.” Poetica 23.3/4 (1991): 295-327. 
von Mücke, Dorothea E. The Practices of the Enlightenment: Aesthetics, Authorship, and the Public. New York: Columbia University Press, 2015.

Webb, Ruth. "The Progymnasmata as Practice." In Education in Greek and Roman Antiquity. Ed. Yun Lee Too. Leiden: Brill, 2001. 289-316.

Wellbery, David E. Lessing's “Laocoon": Semiotics and Aesthetics in the Age of Reason. Cambridge: Cambridge University Press, 1984.

Wellek, René. A History of Modern Criticism: 1750-1950. Vol. 1, The Later Eighteenth Century. London: Jonathan Cape, 1955.

Willer, Stefan. "Orte, Örter, Wörter: Zum locus ab etymologia zwischen Cicero und Derrida." In Rhetorik: Figuration und Performanz; DFG-Symposion 2002. Ed. Jürgen Fohrmann. Stuttgart: J. B. Metzler, 2004. 39-58.

Willer, Stefan. "Was ist ein Beispiel? Versuch über das Exemplarische." In Originalkopie: Praktiken des Sekundären. Ed. Gisela Fehrmann, Erika Linz, Eckhard Schumacher, and Brigitte Weingart. Cologne: DuMont, 2004. 51-65.

Witte, Egbert. Logik ohne Dornen: Die Rezeption von A. G. Baumgartens Ästhetik im Spannungsfeld von logischem Begriff und ästhetischer Anschauung. Hildesheim: Georg Olms, 2000.

Zelle, Carsten. Die doppelte Ästhetik der Moderne: Revisionen des Schönen von Boileau bis Nietzsche. Stuttgart: J. B. Metzler, 1995. 